\title{
SW Sextantis in an excited, low state
}

\author{
P. J. Groot ${ }^{1,2, \star}$, R. G. M. Rutten ${ }^{3}$, and J. van Paradijs ${ }^{1,4}$ \\ 1 Astronomical Institute "Anton Pannekoek" /CHEAF, Kruislaan 403, 1098 SJ, Amsterdam, The Netherlands \\ 2 Harvard-Smithsonian Center for Astrophysics, 60 Garden Street, Cambridge, MA 02138, USA \\ ${ }^{3}$ Isaac Newton Group of Telescopes, Apartado de Correos 321, 38700, Sta Cruz de La Palma, Islas Canarias, \\ Spain \\ 4 Physics Department, UAH, Huntsville, AL 35899, USA
}

Received 24 August 1999 / Accepted 5 December 2000

\begin{abstract}
We present low-resolution spectrophotometric optical observations of the eclipsing nova-like cataclysmic variable SW Sex, the prototype of the SW Sex stars. We observed the system when it was in an unusual low state. The spectrum is characterized by the presence of strong HeII and Civ emission lines as well as the normal single peaked Balmer emission lines. The radial temperature profile of the disk follows the expected $T \propto R^{-3 / 4}$ only in the outer parts and flattens off inside 0.5 times the white dwarf Roche lobe radius. The single peaked emission lines originate in a region above the plane of the disk, at the position of the hot spot.
\end{abstract}

Key words. accretion, accretion disks - binaries: eclipsing - stars: individual: SW Sex - novae, cataclysmic variables

\section{Introduction}

The SW Sex stars are a subclass of the eclipsing novalike (NL) Cataclysmic Variables (CVs). They are classified on a number of spectroscopic features, mainly the single peaked emission lines, the large shifts between spectroscopic conjunction and photometric mid-eclipse, the transient absorption evident in the emission lines around photometric phase 0.5 and the shallow eclipse of the low excitation lines of Hi and He I (see Thorstensen et al. 1991; Warner 1995). Their unusual spectroscopic behaviour has led to intensive studies and a number of possible explanations for the SW Sex "phenomenon": "wind" models (Honeycutt et al. 1986), "overflow" models (Hellier \& Robinson 1994; Hellier 1996; Hellier 1998), "magnetic" models (Williams 1989; magnetic accretion and Horne 1999; magnetic propellors) and "modified standard" models (Dhillon et al. 1997, hereafter DMJ97). Although all of these are capable of explaining a subset of the SW Sex phenonema, none show a conclusive case of explaining all of the features listed above.

SW Sex itself is the prototype of the SW Sex stars and has been the topic of many investigations. It was discovered in the Palomar-Green Survey (Green et al. 1986) as a UV-excess object with high excitation emission lines (Green et al. 1982). Follow-up photometry showed it to

Send offprint requests to: P. J. Groot, e-mail: pgroot@cfa.harvard.edu

* CfA fellow. be a deeply eclipsing system with a $3.24 \mathrm{hr}$ orbit (Penning et al. 1984). We refer to DMJ97 for a recent, more detailed, description on the observational history of SW Sex.

From high-speed broad-band photometry Rutten et al. (1992, hereafter RvPT92) derived a radial dependence of the temperature in the accretion disk that is not only flatter than seen in other systems (see also RvPT92), but also flatter than predicted by standard accretion disk theory (e.g. Frank et al. 1992). This result, combined with the unusual behaviour of the emission lines in SW Sex, prompted us to a study of the accretion disk in SW Sex in more detail. We obtained time series of low-dispersion spectra of SW Sex with the $2.5 \mathrm{~m}$ Isaac Newton Telescope on the island of La Palma.

\section{Data and reduction}

Observations were made on the nights of 25 to 30 March, 1996 using the Intermediate Dispersion Spectrograph (IDS) with the R300V grating and a TEK $1 \mathrm{k} \times 1 \mathrm{k}$ CCD, giving a dispersion of $3.3 \AA$ per pixel over the wavelength range of $3600 \AA$ to $7000 \AA$. In order to minimize slit-losses and optimize the photometric quality of the data the slit was opened to $2^{\prime \prime}$. This set-up resulted in an effective resolution of $8 \AA$. A second star ( $30^{\prime \prime}$ SW of SW Sex) was also placed on the slit to correct for slit-losses. We made time-series with $90 \mathrm{~s}$ integration and $\sim 60 \mathrm{~s}$ dead-time per observation, giving an effective time resolution of $\sim 150 \mathrm{~s}$ or $\sim 1 / 80$ th of an orbital period. Throughout the nights $\mathrm{CuAr}$ calibration spectra were taken for the wavelength 
Table 1. Overview of SW Sex observations March 1996

\begin{tabular}{llll}
\hline Date & Start UT & End UT & No. Exposures \\
\hline $25 / 3 / 96$ & $22: 17$ & $02: 28$ & 62 \\
$26 / 3 / 96$ & $20: 20$ & $01: 55$ & 73 \\
$28 / 3 / 96$ & $23: 17$ & $01: 55$ & 32 \\
$29 / 3 / 96$ & $20: 21$ & $01: 21$ & 70 \\
$30 / 3 / 96$ & $22: 16$ & $02: 20$ & 45 \\
\hline
\end{tabular}

calibration. A total of 282 spectra, covering 9 eclipses, were recorded. An overview of the data is given in Table 1.

The data reduction was carried out using the standard ESO-MIDAS reduction package with additionally written software. All the observations were debiased using the mean of the overscan region on each exposure. A run-averaged flatfield was constructed using internal Tungsten lamp flats for the spectral profile and twilight skyflats for the spatial profile. All spectra were optimally extracted (Horne 1986) and rebinned to a slightly oversampled wavelength-grid with $3 \AA$ wide bins. The wavelength calibration was accurate to $0.15 \AA$. Time and wavelength dependent slit losses were corrected for by applying the variation in the comparison star brightness to SW Sex, while the absolute slit losses were determined from comparing signal strengths of the observations taken with the $2^{\prime \prime}$ wide slit with those taken through a wide, $10^{\prime \prime}$, slit. Flux calibration was done by observing the spectrophotometric standard Feige 34 (Massey et al. 1988) through the same set-up with a $10^{\prime \prime}$ wide slit. Based on Poisson statistics in the extracted spectrum, each wavelength bin was assigned an error, which is propagated to the flux calibrated spectrum. The spectral slope of the comparison star did not correlate with airmass and hence the slit losses due to differential refraction are insignificant.

\section{Eclipse timing}

Phase-folding of the nine observed eclipses, using the ephemeris of DMJ97, gave a phase-offset of minimum light in the photometric light curves of $\sim 0.006$ in phase. We redetermined the ephemeris of SW Sex using the eclipse timings as given in DMJ97 and by making Gaussian fits to our own eclipse light curves (see Table 2 for all eclipse timings). For the existing data we used an error of $110^{-4}$ days on the time of minimum light. For our own data we used an error of $0.510^{-4}$ days, as estimated from the Gaussian fits. A linear regression yields the revised orbital ephemeris given in Eq. (1).

$$
\begin{aligned}
\mathrm{HJD}_{\text {mid_eclipse }}= & 2444339.650574(36) \\
& +0.1349384411(10) \times N .
\end{aligned}
$$

\section{Continuum light-curves}

For the spectrophotometry we used seven of nine observed eclipses. The remaining two (of the night of March 28 and
Table 2. Times of minimum light for SW Sex

\begin{tabular}{llr}
\hline $\begin{array}{l}\text { HJD (mid-eclipse) } \\
(+2440000)\end{array}$ & $\begin{array}{l}\text { Cycle Number } \\
(N)\end{array}$ & $\begin{array}{r}\text { O-C } \\
(\mathrm{s})\end{array}$ \\
\hline 4339.65087 & 0 & -26.33 \\
4340.73055 & 8 & -33.44 \\
4348.82649 & 68 & -23.50 \\
4631.92758 & 2166 & -42.92 \\
4676.86195 & 2499 & -6.74 \\
4721.79636 & 2832 & -12.73 \\
7566.56813 & 23914 & 8.20 \\
7615.41619 & 24276 & 13.33 \\
7615.55065 & 24277 & 28.26 \\
7616.49516 & 24284 & 48.41 \\
7618.51922 & 24299 & 61.44 \\
7619.46374 & 24306 & 39.39 \\
7620.40856 & 24313 & 17.35 \\
7621.48834 & 24321 & 10.24 \\
7622.43257 & 24328 & 30.38 \\
7921.32167 & 26543 & -13.94 \\
7921.45633 & 26544 & 0.98 \\
7950.19842 & 26757 & 17.28 \\
7950.33321 & 26758 & 32.22 \\
8306.30100 & 29396 & 17.89 \\
8306.43599 & 29397 & 32.82 \\
10168.452377 & 43196 & -58.15 \\
10168.586651 & 43197 & -43.22 \\
10169.396799 & 43203 & -38.01 \\
10169.531017 & 43204 & -23.07 \\
10171.554764 & 43219 & 32.14 \\
10172.364589 & 43225 & 37.35 \\
10172.500202 & 43226 & -32.09 \\
10173.443720 & 43233 & 72.42 \\
10173.579514 & 43234 & 2.98 \\
\hline & &
\end{tabular}

Table 3. SW Sex system parameters as used for the eclipse mapping

\begin{tabular}{ll}
\hline Period & $11658.67 \mathrm{~s}$ \\
$M_{\mathrm{WD}}$ & $0.44 M_{\odot}$ \\
$M_{\mathrm{sec}}$ & $0.3 M_{\odot}$ \\
Inclination & $79^{\circ}$ \\
Distance & $290 \mathrm{pc}$ \\
\hline
\end{tabular}

the second eclipse of the night of March 30) were not used because the $\mathrm{S} / \mathrm{N}$ levels of the fainter, secondary star on the slit were too low to make a reliable slit correction. This leaves a total of 220 spectra of SW Sex to be used.

From the spectrophotometry light curves can be extracted at any of the observed wavelength regions. Figure 1 shows the continuum light curves between $\lambda \lambda 4200-4240$, 5300-5340 and 6720-6760 $\AA$ as examples. Apart from the eclipse, the light curves show the presence of an orbital hump that disappears towards longer wavelenghts.

Comparison of the continuum light curves with previously published data shows a similar amount of eclipse profile asymmetry as in DMJ97, Penning et al. (1984) and Ashoka et al. (1994) for the $\lambda$ 4200-4240 $\AA$ light curve.

The elementary eclipse-mapping program, as will be applied in Sect. 5, assumes that all variation in the light 

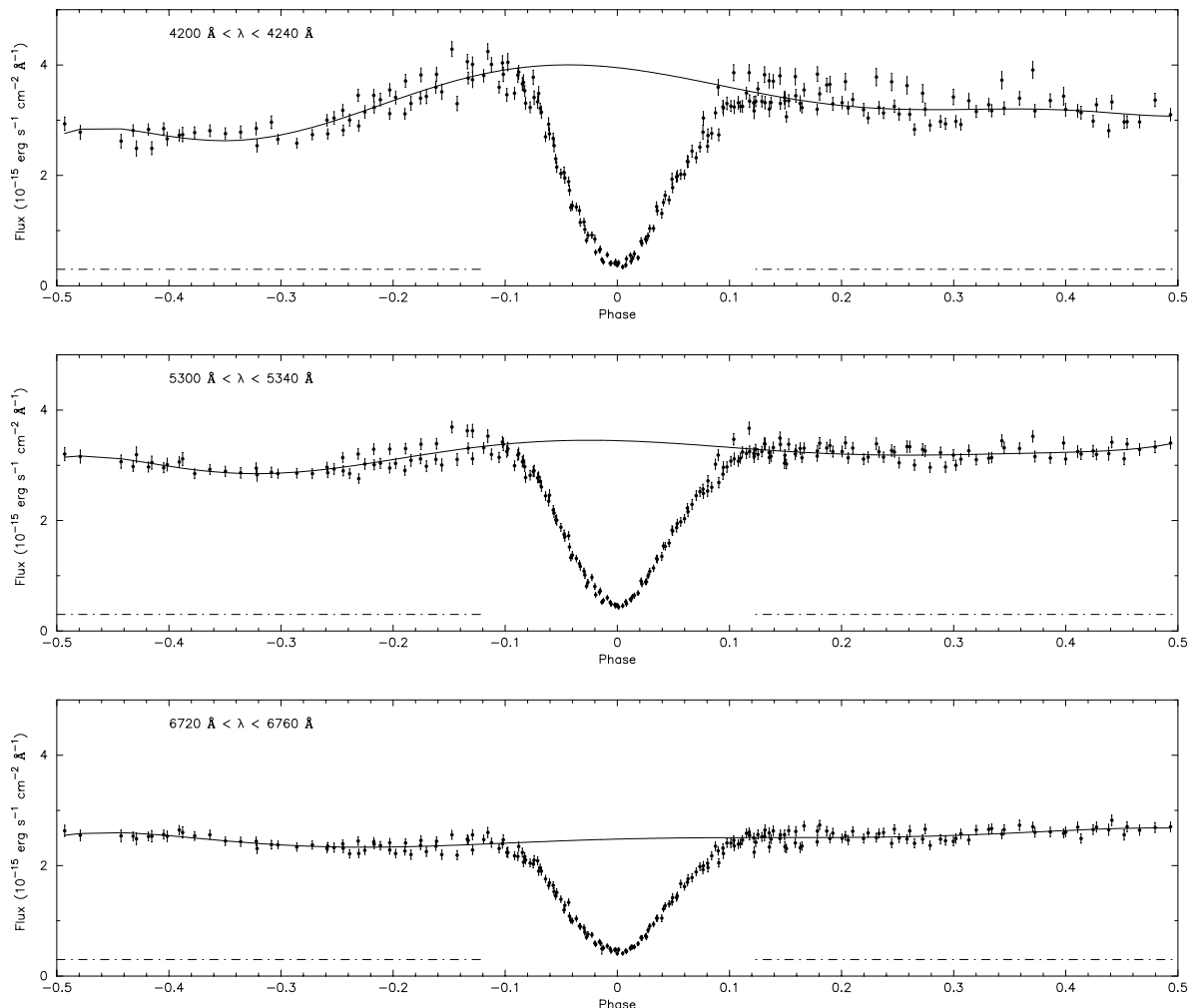

Fig. 1. The spectrophotometric light curve of SW Sex between $4200 \AA<\lambda<4240 \AA$ (top), $5300 \AA<\lambda<5340 \AA$ (center), and $6720 \AA<\lambda<6760 \AA$ (bottom). A pronounced orbital hump is seen in the blue light curve, which gradually diminishes and is gone at the reddest wavelengths. The 7 th order polynomial fits to the light curves outside eclipse are shown as the full lines

curve occurs during eclipse. We therefore need to apply a correction to take away any variations outside elipse. We have applied a polynomial fit to the phases smaller than -0.12 and larger than 0.12 . Trial fits with different order polynomials showed that a 7 th order polynomial returned the smallest residuals, independent of wavelength.

\section{Spectral eclipse mapping}

Eclipse mapping uses the information contained in the shape of the eclipse light curve to reconstruct the light distribution on the accretion disk. For a general description of the method we refer to Horne (1985). We have used the same program as was used by Rutten et al. (1994) for the analysis of UX UMa, which is a maximum-entropy based optimization routine, as described in Horne (1985; see also Gull \& Skilling 1983). For the reconstruction of the accretion disk in SW Sex we used the basic, thin, flat disk approach, as was also used in Rutten et al. (1992), who also show that the temperature profile is not affected by the assumptions about the shape or thickness of the disk. No correction for interstellar extinction was deemed necessary since IUE spectra show no presence of the $2200 \AA$ bump (RvPT92).

\subsection{Light curve selection and preparation}

We have split the total wavelength range covered by our spectra $(3650-7000 \AA)$ in 79 wavelength bins, each $40 \AA$ wide, with the exception of the spectral lines, which were taken as one bin each (with variable width).

The errors which were assigned to each spectral bin on the basis of Poisson statistics were propagated to the light curves. SW Sex was very stable during the six nights of our observations and no matching of the individual light curves was required. A small amount of random variation is seen in the blue part of the spectrum, but this diminishes towards the red, and is therefore most likely dominated by intrinsic flickering. These variations will cause the $\chi^{2}$-based eclipse mapping routine to reconstruct spurious fine structure in the resulting map. To avoid this we have increased the formal errors by up to $40 \%$ for the bluest wavelengths. In the red part of the spectrum this adjustment was not more than $10 \%$.

For the reconstructions as presented here, we used a $51 \times 51$ pixel map, with the phase interval $-0.20<\varphi<$ 0.20 as input. We have set no a priori limit to the size of the accretion disk. The light curve zero-point was taken as a free parameter in the fit to account for any uneclipsed light (RvPT92).

\subsection{Reconstructed intensity distribution}

In Fig. 2 we show the resulting eclipse map in the middle part of our spectrum. The map shows a clear dominance of the white dwarf and surroundings, which is reconstructed as the bright region in the middle. On the scale used here 
the size of the WD corresponds to $\sim \frac{1}{4}$ pixel. We see that the emission is dominated by the WD and surroundings. In the blue maps a hot-spot contribution is seen, whose strength diminishes towards the red, consistent with the light curve behaviour.

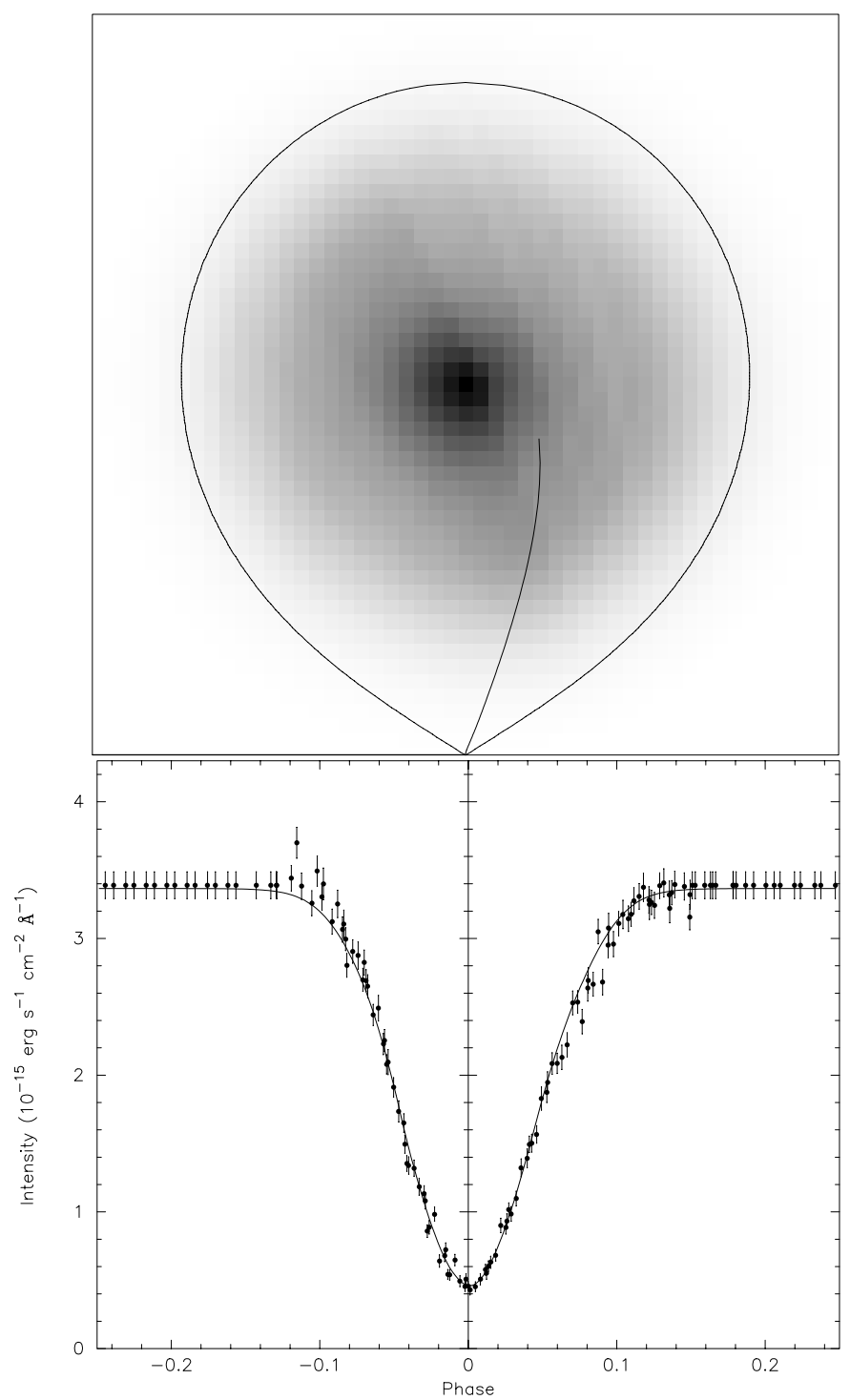

Fig. 2. The reconstructed accretion disk for the wavelength region between $5490 \AA$ and $5530 \AA$ (top). The bottom panel shows the input (dots) and reconstructed (line) lightcurves

\subsection{Size of the accretion disk}

To measure the size of the accretion disk we use the same measure as in RvPT92, namely the point where the intensity has fallen to $10 \%$ of the central intensity. At $4060 \AA$ we measure $R_{\mathrm{d}} \simeq 0.7 R_{\mathrm{L}_{1}}$, where $R_{\mathrm{L}_{1}}$ is the distance between the inner Lagrangian point $\left(L_{1}\right)$ and the white dwarf. At azimuth angles that are pointing towards the hot-spot region (as seen from the WD) the apparent size of the disk reaches up to $0.9 R_{\mathrm{L}_{1}}$. At $5510 \AA$ the disk reaches up to 0.65-0.7 $R_{\mathrm{L}_{1}}$, slightly depending on azimuth angle, and at

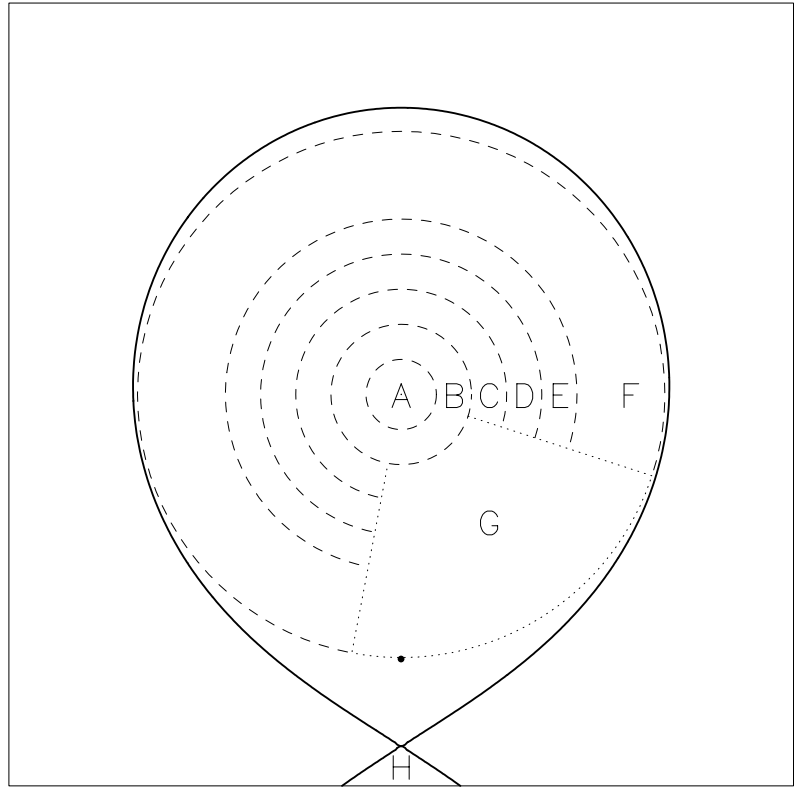

Fig. 3. To reconstruct the accretion disk spectrum the WD Roche-lobe has been divided in 7 segments, labeled "A" through "G" and located, respectively, between 0-0.1, 0.1-0.2, $0.2-0.3,0.3-0.4,0.4-0.5,0.5-0.75 R_{\mathrm{L}_{1}}$. The segments further out than $0.2 R_{\mathrm{L}_{1}}$ are split up between the part between phase 0.03 and 0.8 (section "F") and the hot-spot part between phase 0.8 and 0.03 (section "G"). The component of uneclipsed light is represented by segment " $\mathrm{H}$ ", here tentatively located at the secondary

$6350 \AA$ the $10 \%$ level is reached at $0.75 R_{\mathrm{L}_{1}}$, independent of azimuth angle. The azimuth dependence with wavelength reflects the diminishing influence of the hot-spot in SW Sex. The disk radii measured here are slightly larger than measured by RvPT92.

\subsection{Accretion disk spectrum}

The reconstructed intensity maps allow us to deduce the spectrum at any given point in the disk. Since the eclipsemapping procedure smooths the disk in the azimuthal direction and since we are mainly concerned with the radial profile of the disk, we have used a set of concentric rings (shown in Fig. 3) and a hot-spot region. The reconstructed spectra are shown in Fig. 4. There is spectral variation in the radial direction, going from a relatively blue spectrum in the middle of the disk to a redder spectrum near the edges of the disk. The hot-spot region ("G") is clearly much bluer than the other section of the outer ring ("F"). Comparing the spectral evolution seen here with the one that was found in UX UMa by Rutten et al. (1994), we see that the changes in SW Sex are much more moderate and less pronounced than in UX UMa, whose temperature profile does follow the $T \propto R^{\frac{-3}{4}}$ relation as predicted by the theory of steady state accretion disks (see e.g. Frank et al. 1992). 


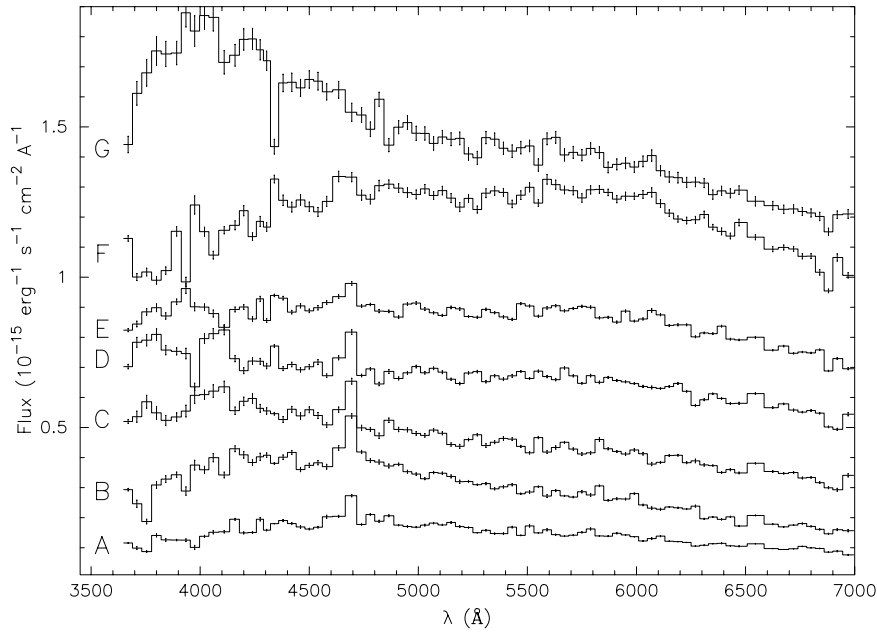

Fig. 4. Reconstructed accretion disk spectrum in the regions A through $\mathrm{G}$ as indicated in Fig. 3. To separate the spectra from each other shifts have been applied of respectively -0.1 , $0.05,0.25,0.46,0.55,0.85$ for the regions A through G. Fluxes have been calculated as the summed flux in each segment

\subsection{Uneclipsed light}

Any uneclipsed component to the light curve could produce spurious results in the final eclipse map (see RvPT92). For that reason the uneclipsed light component was incorporated as a free parameter in the optimization routine. The spectrum of this uneclipsed component is shown in Fig. 5. It prominently shows the Balmer series in emission. A well known feature of SW Sex systems is the partial eclipse of the Balmer lines and the occurence of these lines in emission in the uneclipsed component is consistent with this. The uneclipsed component is most likely to emanate from either the secondary star or from outside the orbital plane. The secondary star in SW Sex has not been detected. The slope of the uneclipsed spectrum as reconstructed here is indicative of a late M-type secondary, which suggests (some of) the light could represent the secondary.

\subsection{Accretion disk temperature distribution}

We reconstruct a temperature distribution on the accretion disk by making Planck curve fits to the spectrum at each pixel on the disk, assuming the complete disk is optically thick and radiating as a blackbody. The flux received on Earth is:

$f=\frac{N A \cos i \sum_{j=1}^{N} \sigma T_{j}^{4}}{4 \pi d^{2}}$

with $N$ the number of pixels on the disk, $A \cos i$, the projected size of each pixel, $T_{j}$ the effective temperature of each pixel and $d$ the distance to the source.

The distance to SW Sex is not well known. An uncertain value of $250 \mathrm{pc}$ is quoted by Patterson (1984), derived from the $\mathrm{H} \beta$ equivalent width and the continuum shape. RvPT92 derived 450 pc from blackbody fits to the inner

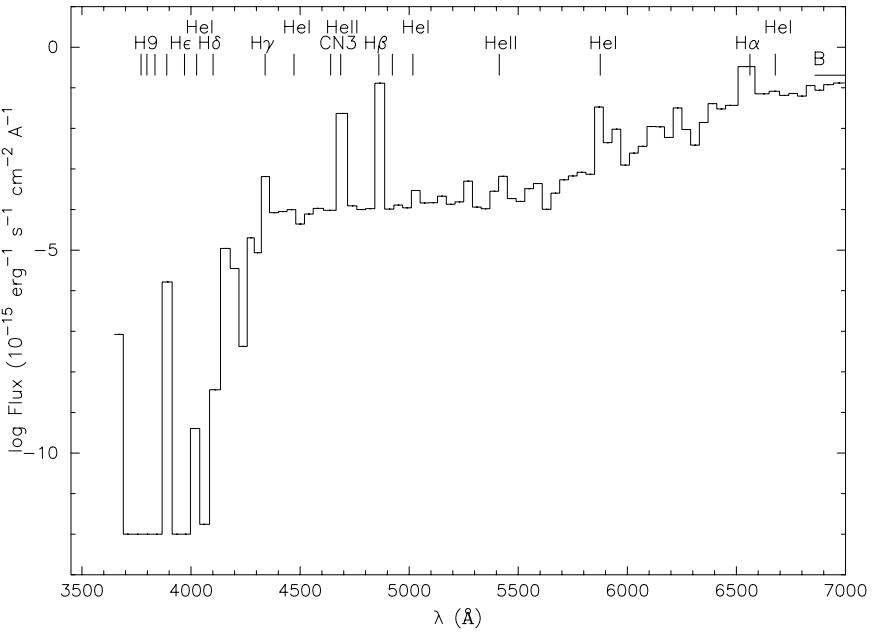

Fig. 5. The reconstructed spectrum of the uneclipsed light component. The continuum levels $<4200 \AA$ are computational noise

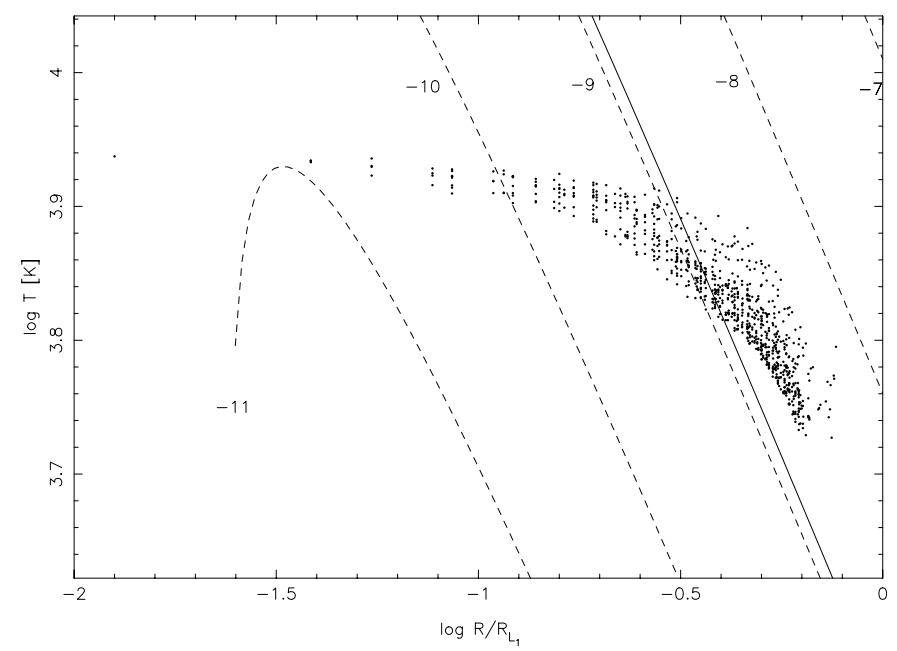

Fig. 6. The derived $\dot{M}$ profile as function of the radial distance from the white dwarf. The solid line shows the averaged fitted mass-transfer rate. The dashed lines show the expected profile on the basis of standard accretion disk theory in units of $10 \mathrm{log}$ of the mass transfer rate in $M_{\odot} \mathrm{yr}^{-1}$ (i.e. "-10" is $\left.10^{-10} M_{\odot} \mathrm{yr}^{-1}\right)$

accretion disk in their four colour photometry and the absence of any feature of the secondary. Including the distance as a free parameter in our blackbody fits, we derive a distance to SW Sex of $290 \mathrm{pc}$. Using the value of $450 \mathrm{pc}$ as found by RvPT92 gave clearly incorrect blackbody fits at all points in the disk. Using $290 \mathrm{pc}$ for the distance, we obtain a temperature map of the accretion disk of SW Sex, of which the radial cut is shown in Fig. 6. Note that any uncertainty in the distance affects the temperatures but less so the radial dependence.

The current results confirm those of RvPT92 that the radial temperature dependence does not follow the standard, steady state prediction, but deviates at radii $<0.5$ $R_{\mathrm{L}_{1}}$. Although Smak (1994) argued that the flat temperature profile is due to obscuration of the inner parts of the 


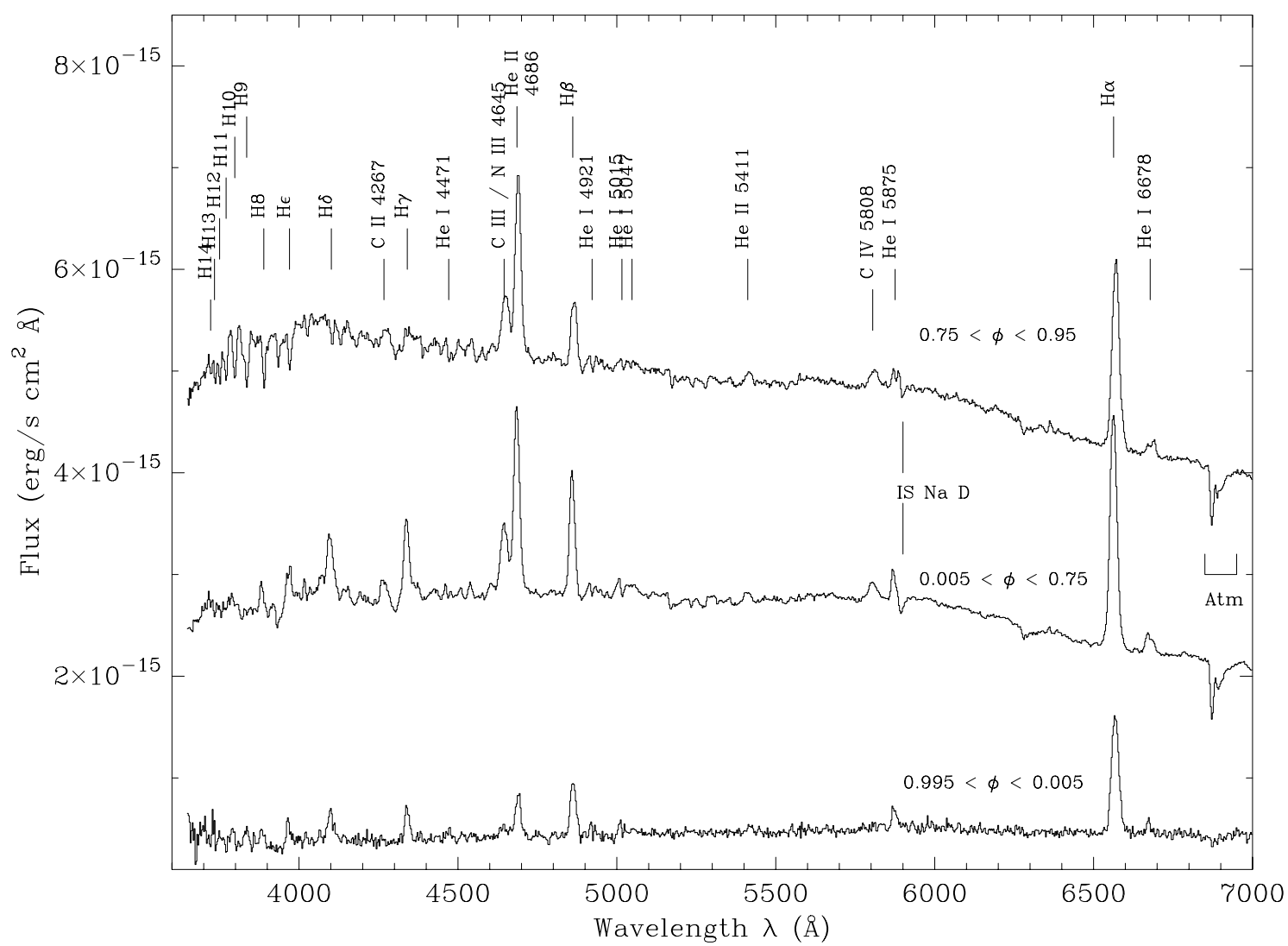

Fig. 7. The average spectrum of SW Sex at phase intervals $0.995<\varphi<0.005$ (bottom), $0.005<\varphi<0.75$ (middle) and $0.75<\varphi<0.95$ (top, offset by $+2.010^{-15} \mathrm{erg} \mathrm{s}^{-1} \mathrm{~cm}^{-2} \AA^{-1}$ ). Major lines are indicated

disk, this would require a disk flare angle of more than $11^{\circ}$, which appears rather large (see also Rutten 1998).

\section{Spectral line behaviour}

In the spectral eclipse mapping we have used the spectral lines as single wavelength bins. We will now investigate in further detail the spectral line behaviour.

\subsection{Average spectrum}

Before we "zoom in" on the spectral lines we first show the average spectrum of SW Sex during our run in Fig. 7.

The out-of-eclipse $(0.005<\varphi<0.75)$ shape of the spectrum of SW Sex (Fig. 7, middle curve) is typical for SW Sex stars: strong single peaked Balmer, HeII and NIII/CIII emission on top of a flat or slightly blue continuum. However, there are a number of marked differences with previous spectroscopic studies of SW Sex (Green et al. 1982; Penning et al. 1984; Williams 1989 and DMJ97):

- The ratio of HeII $\lambda 4686$ over $\mathrm{H} \beta$ is larger than unity;

- The HeI lines are unusually weak;

- The presence of CIV $\lambda 5805$ and HeII $\lambda 5411$.

These three unusual features show that the emission lines are formed in a site with a higher than usual ionization level.
Furthermore, the system is approximately $1.2 \mathrm{mag}$ fainter than when observed by Penning et al. (1984), Honeycutt et al. (1986) and RvPT92. The depth of the eclipses (see Sect. 4) is not significantly different (1.9-2.0 mag) from previous epochs.

\subsection{Multiple emission components}

We have binned the data in 50 phase bins and subtracted the underlying continuum by making a linear fit to adjacent wavelengths. The trailed spectra of the main emission lines are shown in Fig. 8.

It is clear that all lines show a complex behaviour, with multiple emission and absorption components present. The low excitation Balmer lines $(\mathrm{H} \alpha, \mathrm{H} \beta)$ and HeII $\lambda 4686$ are dominated by an S-wave, with variable emission strength. However, the higher excitation Balmer lines, the HeI lines and the Cir $\lambda 4267$ line have multiple components. Although the low-resolution of our data defeats an unambigious interpretation, the behaviour of the HeI $\lambda 6678$ and $\lambda 5875$ lines suggests two separate S-waves that intertwine. Our preference for this, over the explanation of one S-wave with variable absorption components superposed on it, is given by the difference in the emission profile width at phase 0.4 compared to phase 0.1 . The HeI $\lambda 5875$ is badly affected by the interstellar $\mathrm{Na} \mathrm{D}$ doublet at $\lambda 5890 \AA$. 


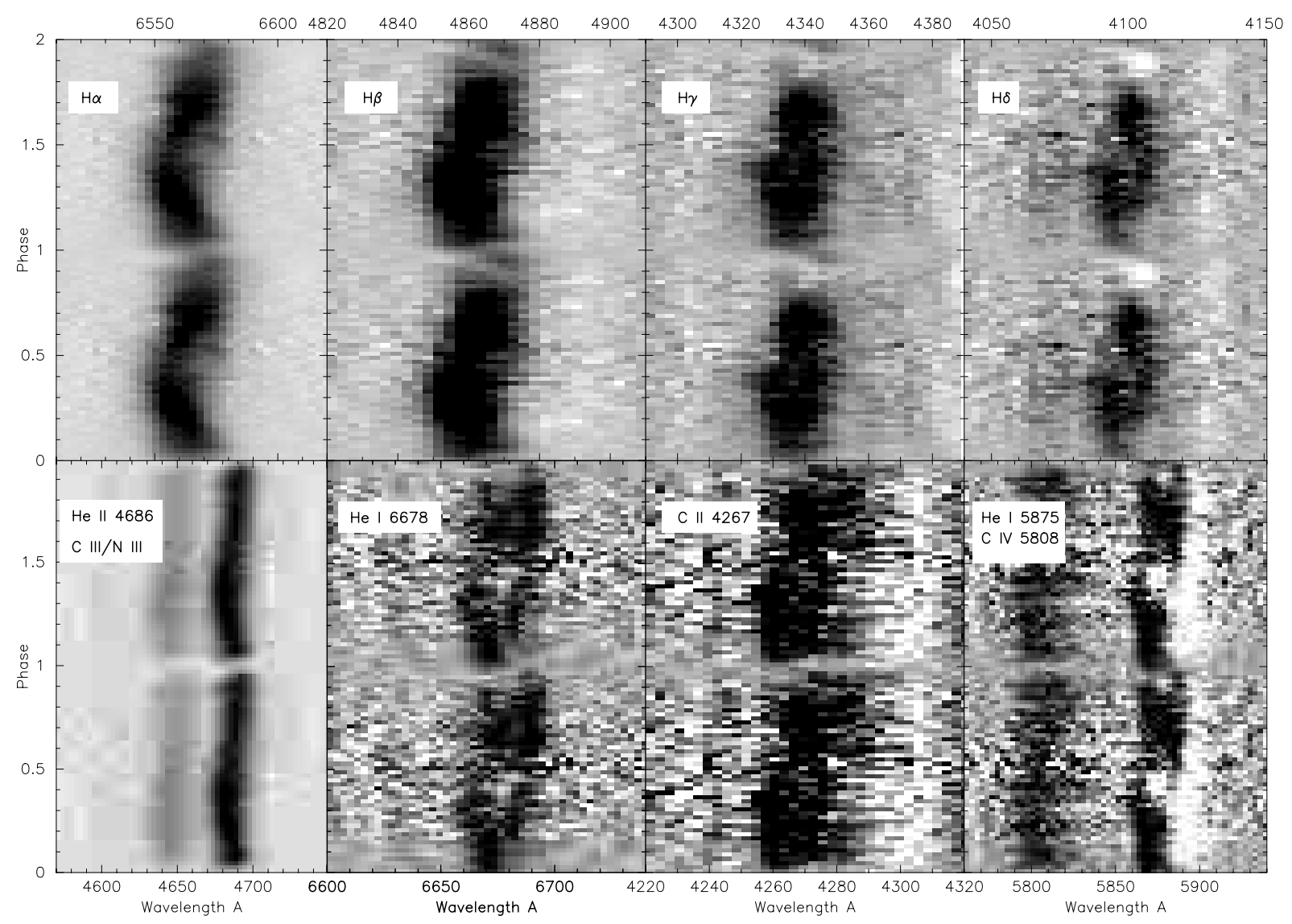

Fig. 8. Trailed spectra of $\mathrm{H} \alpha, \mathrm{H} \beta, \mathrm{H} \gamma$ and $\mathrm{H} \delta$ (top, left to right), and HeII $\lambda 4686$ / CiıI, Niı $\lambda 4645$, HeI $\lambda 6678$, Ciı $\lambda 4267$ and HeI $\lambda 5875$ and Civ $\lambda 5808$ (bottom, left to right). The HeI $\lambda 5875$ line is badly affected by the NaI doublet next to it

\subsection{Radial-velocity curves of the main S-wave component}

The radial-velocity curves of the main component visible in $\mathrm{H} \alpha$ and HeII $\lambda 4686$ are shown in Fig. 9. These were determined by fitting a single Gaussian to the line profiles at phase intervals of 0.02 . The Gaussian fit is effectively determined by the flanks of the emission lines. In both lines the main component has its red-to-blue crossing at $\varphi=0.09$. This indicates that the emission is not symmetrically centered on the $\mathrm{WD}$, since in that case the red-toblue crossing would coincide with superior conjunction of the white dwarf, i.e. at $\varphi=0$. This phase lag of the emission lines is a well known characteristic of SW Sex stars (see e.g. Thorstensen et al. 1991). The phase lags are in good agreement with the results from DMJ97.

The 0.09 phase offset indicates that the emission site is not on the line of centers, between the center-of-mass $(\mathrm{CoM})$ and the white-dwarf, but at a slight angle to it. (See Fig. 15 for a graphic summary). The identical phase lag and amplitude of the radial velocity curve indicate that both $\mathrm{H} \alpha$ as well as HeII $\lambda 4686$ originate in the same location.

If we take the average of the measured values for the two spectral lines as the (resultant) velocity vector of the emitting material and correct for the orbital inclination $\left(i=79^{\circ}\right)$, we get $v_{\text {res }}=230 \mathrm{~km} \mathrm{~s}^{-1}$ for the emitting material.

Both radial velocity curves show a rotational disturbance around mid-eclipse. This rotational disturbance is caused by the progressive obscuration of first the blueshifted gas, and later the red-shifted gas in the rotating accretion disk. The occurence of a rotational disturbance shows that part of the emission originates in the accretion disk.

For both lines maximum redshift of the rotational disturbance occurs at $\varphi \sim 0.95$, which coincides with the moment of ingress of the white dwarf and the hot-spot region. Maximum blue-shift, however, is reached for both lines at phase, $\varphi \sim 0.03$. This is too early to be explained by an emission site in the plane of the disk if Roche geometry holds. This indicates that, at $\varphi \sim 0.03$, the main emission site becomes visible again and dominates the emission profile over the disk component.

The observed (phase-lag, radial velocity amplitude)pair is the resultant of a vector addition of two components: the orbital velocity, which is directed perpendicular to the line joining the emission site and the center-ofmass, and the flow velocity of the material in the emission site around the white dwarf. If we know the position of the emission site, we can calculate the orbital velocity of material at that particular distance of the WD and since we also know the resultant velocity vector from the radial 

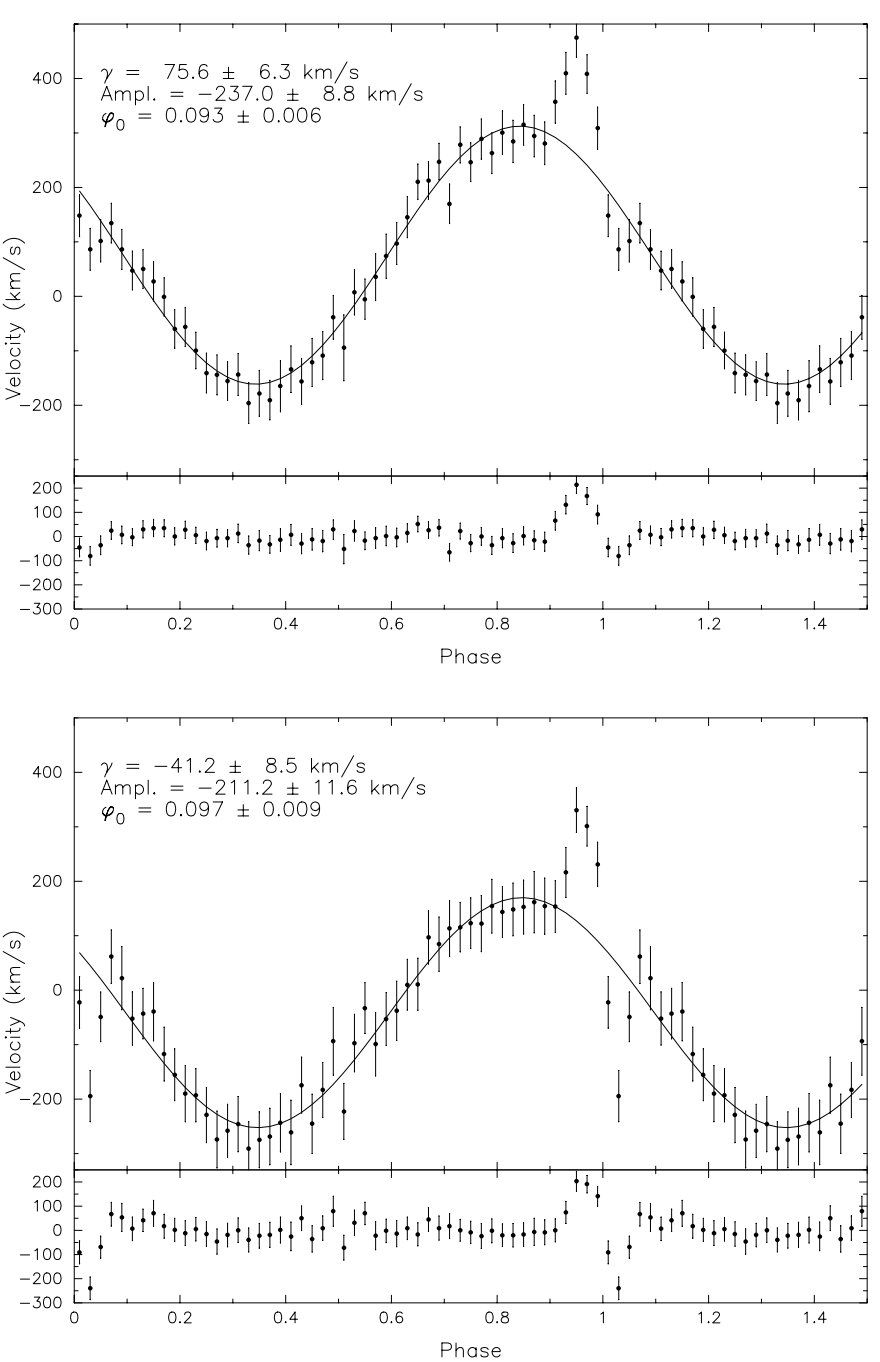

Fig. 9. Radial velocity curve of $\mathrm{H} \alpha$ (top) and HeII $\lambda 4686$ (bottom). Full lines show the best sinusoidal fit to the data. Residuals are given in the lower panels

velocity curve, we can determine the true gas-flow velocity of the material around the WD (see Sect. 8.1).

We have not attempted to obtain radial velocity curves for any of the other lines presented in Fig. 8 because of the multicomponent nature of these lines, as presented and discussed in Sect. 6.2.

\subsection{Line light-curves}

Line light-curves for the lines presented in Sect. 6.2 were extracted by summing all data in the relevant wavelength regions and subtracting from these a linear fit to the adjacent continuum (Fig. 10). As was already evident in the trailed spectra (Fig. 8), the line light-curves show substantial variations outside eclipse. The distorted profiles are very similar to those that have been seen before in SW Sex (DMJ97).

The light curve of HeII $\lambda 4686$ is very similar to that of the continuum, and is dominated by the eclipse. In the other light curves presented in Fig. 10 only $\mathrm{H} \alpha$ and
CII $\lambda 4267$ show the presence of an eclipse. The Balmer series light curves are dominated by a broad V-shaped feature that is centered on $\varphi \sim 0.95$.

\section{The hot spot}

\subsection{Position of the hot-spot}

To determine the position of the hot-spot we have added up the eclipse maps between $3800 \AA$ and $4140 \AA$, where the hot-spot continuum spectrum is the brightest. This sum map is then divided by a map at red wavelengths to obtain the ratio of the two. This ratio map is shown in Fig. 11 and brings out the location of the hot-spot at $r_{\mathrm{hs}}=0.4-0.6 \quad R_{\mathrm{L}_{1}}$ and azimuth angles $0.93<\varphi<1.0$. The azimuthal extent is somewhat uncertain because of the smearing in the azimuthal direction as applied in the eclipse mapping method.

\subsection{Hot-spot absorption line spectrum}

During the phases that the hot-spot is visible, $0.75<\varphi<$ 0.95 , the blue spectral shape changes dramatically with respect to the spectrum during the rest of the orbit. The higher Balmer lines change from general emission between phases $0.005<\varphi<0.75$ to absorption, visible up to H14, as shown in Fig. 12. At the same time, the higher excitation lines of HeII $\lambda 4686$, Cir $\lambda 4267$ and the CIII/NIII $\lambda 4645$ complex do not change in strength compared to the continuum and each other.

The ensemble of absorption lines as present in the hot-spot spectrum can be identified as those occuring in an early B-type spectrum (e.g. the B0 type star HD 77581/Vela X-1; Van Kerkwijk et al. 1995). Typical temperatures in stellar photospheres of B0-type stars range between $19000 \mathrm{~K}<T_{\text {abs }}<25000 \mathrm{~K}$, depending on local gravity. Since we expect a relatively low local gravity in the hot-spot region $\left(g \sim 0.1 g_{\odot}\right.$, comparable to a giant's photospheric gravity; Marsh 1988), the temperature will be on the lower end of this range. Deducing a more exact determination of the temperature of this absorption region is compounded by two factors: the low-resolution of the data and the fact that most of the absorption lines that are normally used for spectral classification of OBtype stars (HeI, HeII, CII, CIII) are in emission in SW Sex. However, we can set a tentative lower limit of $\sim 15000 \mathrm{~K}$ (corresponding to a B2 spectral type), from the fact that the Sirv $\lambda 4089$ line is visible and the MgII $\lambda 4481$ line is not visible.

\subsection{Absorption line spectrum light curve}

For this we have constructed the trailed spectrum and light curve of $\mathrm{H} 8$ (Fig. 13). We have chosen this line because it is clearly present in the absorption line spectrum of Fig. 12 and is relatively well isolated, which allows a reliable continuum subtraction. We see in Fig. 13 that the light curve of $\mathrm{H} 8$ does not resemble that of the 


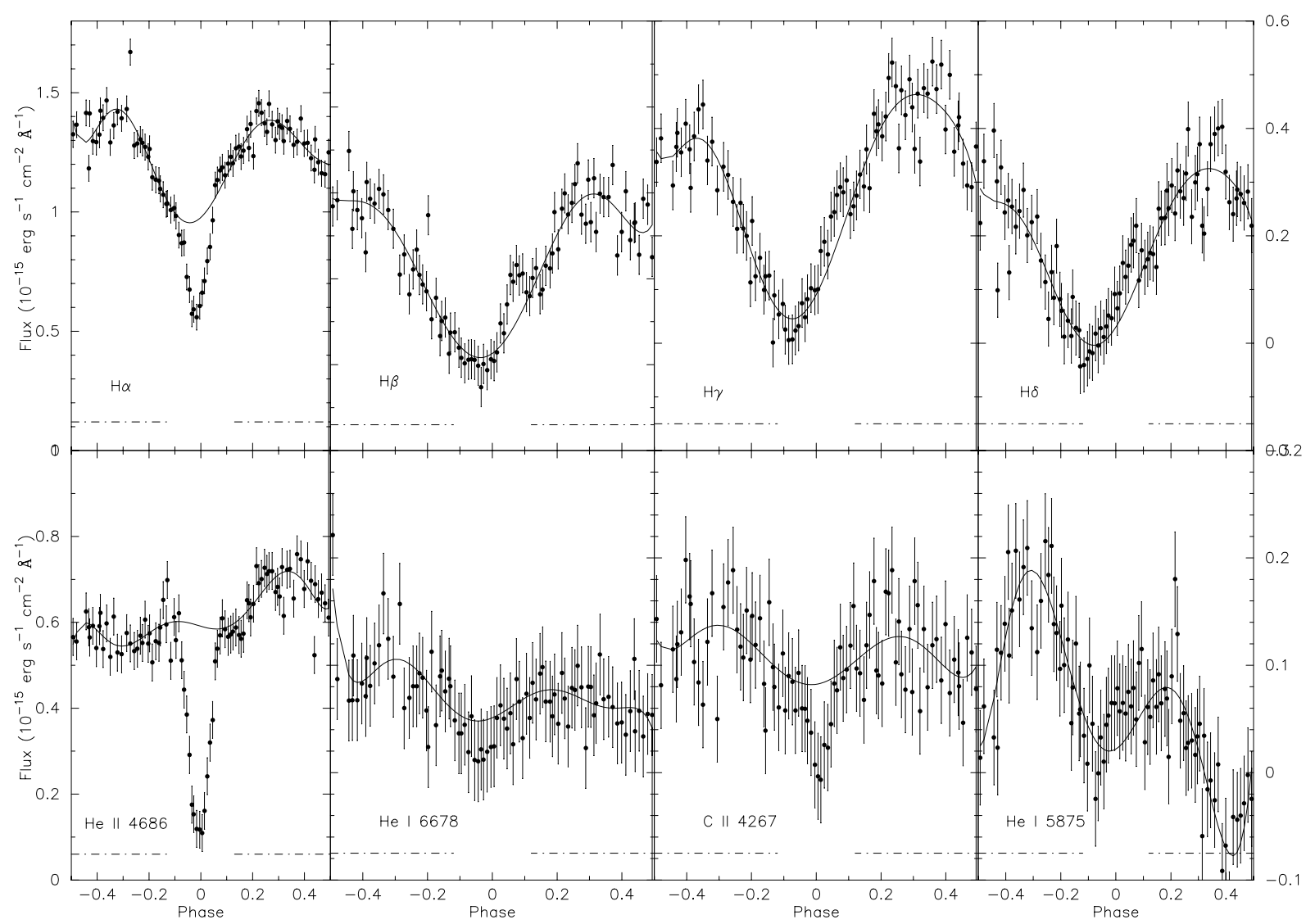

Fig. 10. The spectrophotometric light curves of the lines presented in Fig. 8. Data have been rebinned to 100 phasebins to allow for meaningfull continuum subtraction. The solid line shows the 7 th order polynomial fit to the phase intervals outside -0.12 and 0.12 (indicated by the dashed-dotted line). The scale for $\mathrm{H} \beta$ is 0 to $110^{-15} \mathrm{erg} \mathrm{s}^{-1} \mathrm{~cm}^{-2} \AA^{-1}$, for $\mathrm{H} \gamma$ and $\mathrm{H} \delta$ it is given on the top right hand side of the plot, for HeI $\lambda 6678$, Ci $\lambda 4267$ and HeI $\lambda 5875$ it is given on the bottom right side of the figure

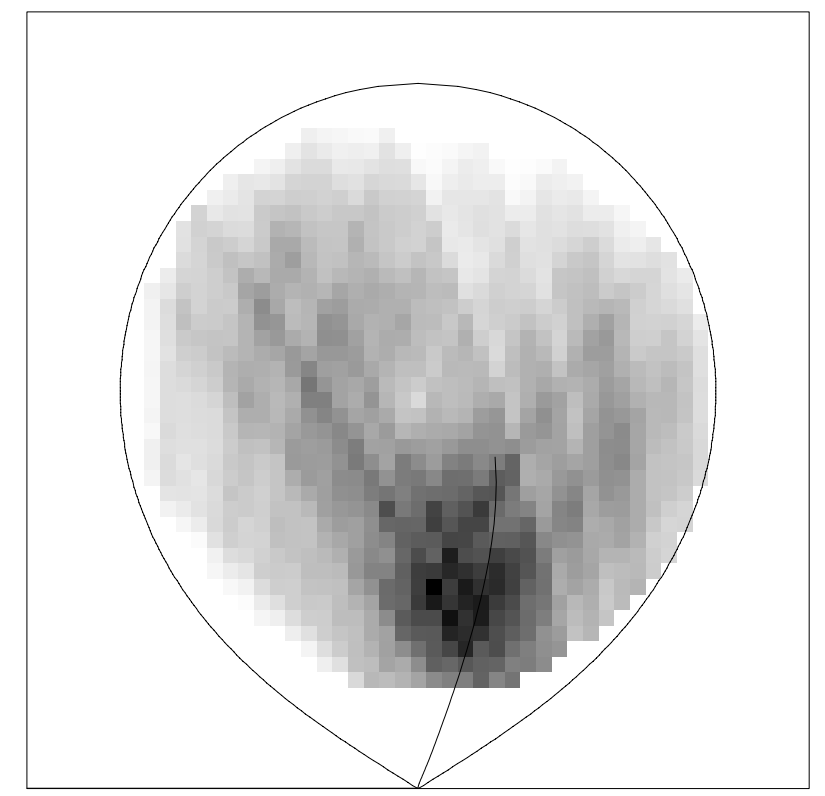

Fig. 11. The reconstructed hot-spot map between $3800 \AA$ and $4140 \AA$. The map was made by summing the maps in this wavelength region and dividing it by the map at $6350 \AA$ to obtain solely the hot-spot part. The map shows that the hotspot is located at $0.4-0.6 R_{\mathrm{L}_{1}}$, and has azimuthal extent of $0.93<\varphi<1.0$ in phase lower Balmer series. Instead of a wide V-shape, it is almost U-shaped and the absorption only extends from $0.82<\varphi<0.94$.

\subsection{The hot spot continuum spectrum}

Although we have deduced in the previous paragraph that the hot-spot region must contain gas at temperatures exceeding $15000 \mathrm{~K}$, this appears to be at odds with the eclipse mapping results given in Sect. 5.6, where we see that the temperature in the hot-spot region does not exceed $10000 \mathrm{~K}$. Here we will investigate whether the temperature derived from the absorption spectrum, $T_{\text {abs }}$ is the same as that derived from the hot-spot continuum radiation, $T_{\text {spot }}$.

To this end we take the spectrum as reconstructed in the spectral eclipse mapping in region "G" in Fig. 3. Since this region will also contain parts of the accretion disk which are not affected by the accretion stream impact, and since we expect the hot-spot region to be the hottest part of this region, we will concentrate on the blue part of the spectrum $(\lambda<5000 \AA)$ to derive the hot-spot continuum temperature.

We have used the spectral catalogue of Jacoby et al. (1984) to compare the hot-spot region "G" spectrum with that of a wide range of spectral types and luminosity 


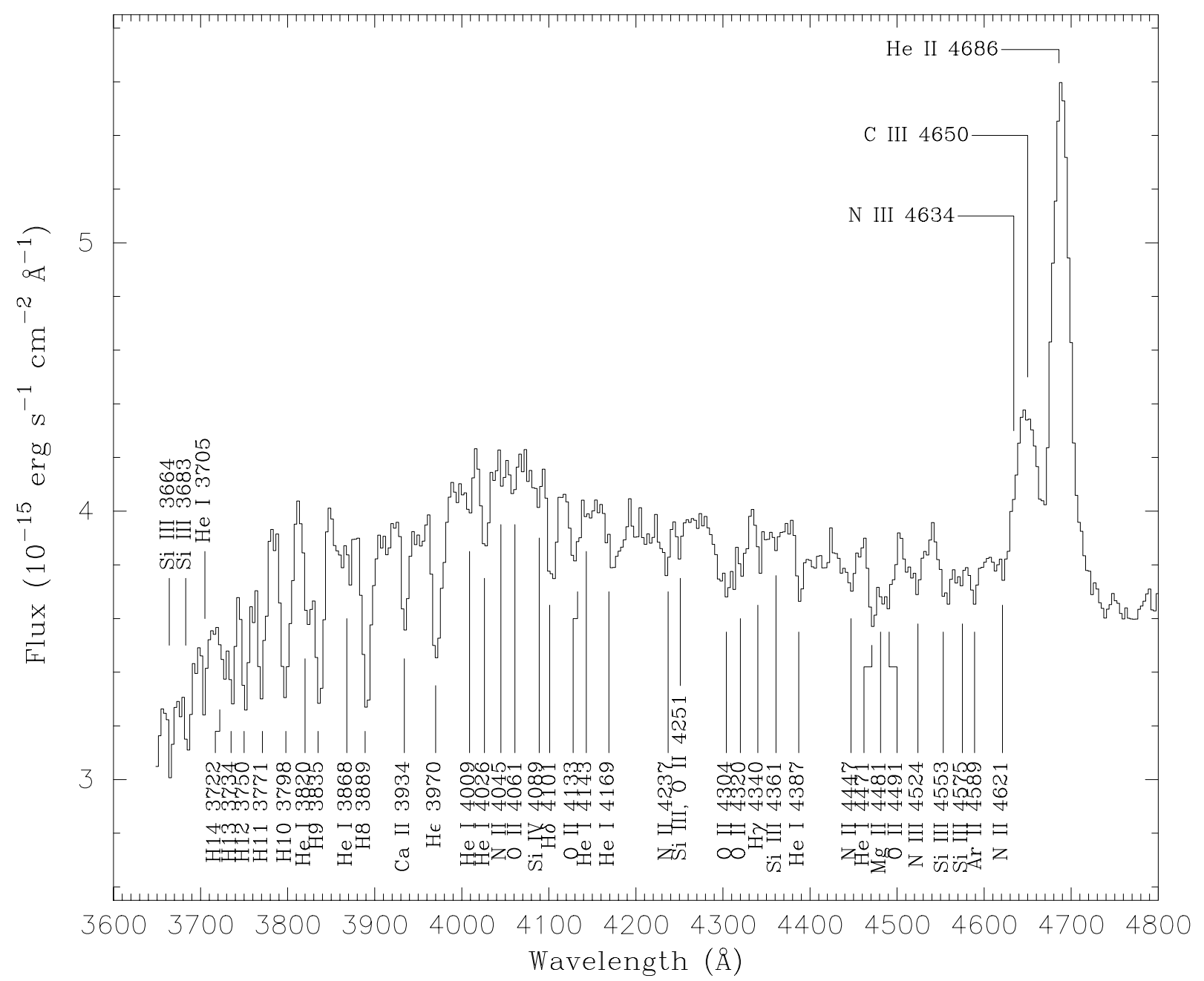

Fig. 12. The blue part of the average spectrum at $0.75<\varphi<0.95$. The absorption line identifications show the spectrum to be identical to a B0-type spectrum, indicating absorbing material temperatures of $19000 \mathrm{~K}<T_{\text {abs }}<25000 \mathrm{~K}$. Line identifications have been taken from Van Kerkwijk et al. (1995)

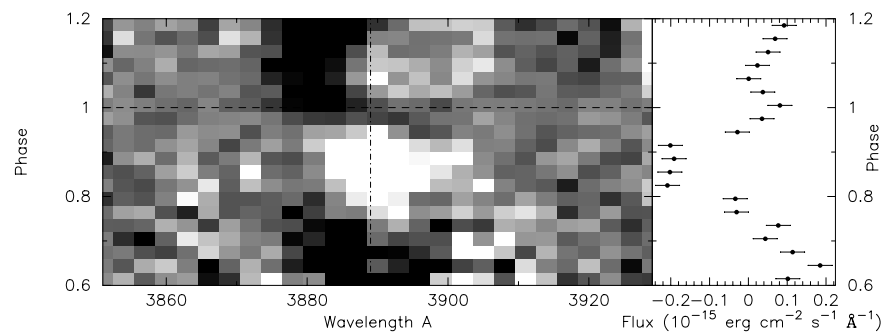

Fig. 13. Trailed spectrogram of the H8 ( $\lambda 3889)$ line (left) and its lightcurve (right) from phase 0.6 to 1.2. The horizontal dashed line shows mid-eclipse according to Eq. (1), the vertical dashed line is the central wavelength

classes. As mentioned above we expect the effective gravities in the hot-spot region to be similar to a giant's atmosphere, and we have therefore restricted ourselves to spectra of luminosty class III, although the differences in luminosity class are almost indistinguishable at the low resolution of the reconstructed hot-spot spectrum.

In Fig. 14 we compare our hot-spot spectrum (solid line) with the B8III spectrum of HD 28696, rebinned to the same spectral resolution and scaled to the same flux (thick dashed line). Also shown are a B5III and an A3III spectrum. The B5III spectrum underestimates the Balmer jump considerably, and hence the hot-spot continuum must be cooler than B5III $\left(T_{\text {spot }}<12000 \mathrm{~K}\right)$.

Consequently, the gas, which we see in the absorption line spectrum as presented in Sect. 7.2 is of a higher temperature (with a lower limit of $T_{\text {abs }}>15000 \mathrm{~K}$ ) than the gas that emits the hot-spot continuum radiation, for which we can put a very conservative upper limit of $T_{\text {spot }}<12000 \mathrm{~K}$. If the Balmer jump in our hot spot continuum spectrum were to be underestimated (e.g. due to emission in the higher Balmer lines) the discrepancy between the temperature deduced from the absorption lines and the continuum would become even worse.

\section{Discussion}

\subsection{Emission region velocities}

With the localization of the hot spot from the eclipse mapping and the $\mathrm{H} \alpha$ and HeII $\lambda 4686$ radial velocity curves we 


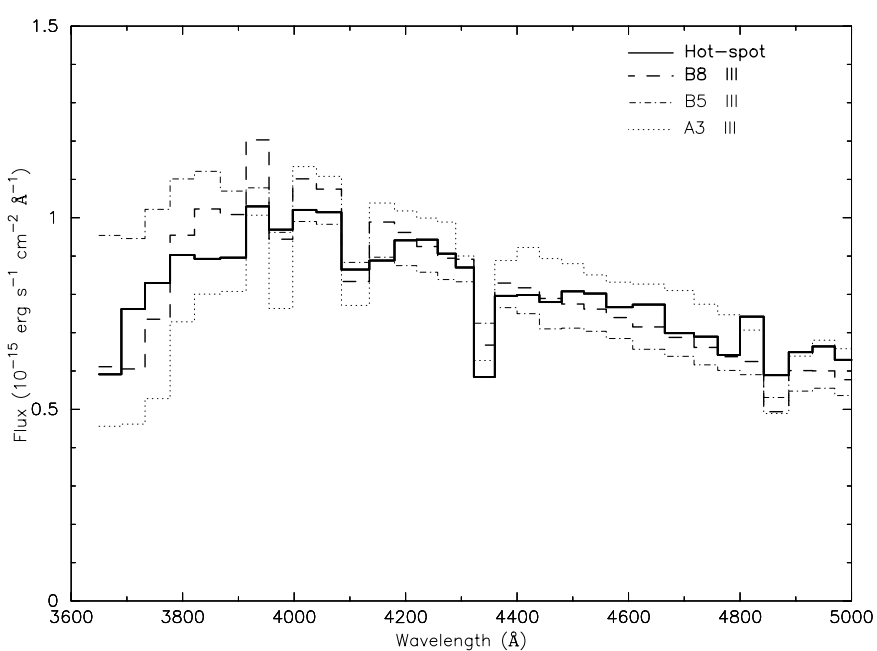

Fig. 14. The reconstructed hot-spot spectrum, compared with three standard star spectra from Jacoby et al. (1984)

can reconstruct the velocity vector of the material in the line emission region. In Fig. 15 we show a schematic picture of the white dwarf Roche lobe with the velocity vectors included. The orbital velocity $\left(v_{\text {orb }}\right)$ at the position of the hot spot, using the system parameters of SW Sex as listed in Table 3, is $60 \mathrm{~km} \mathrm{~s}^{-1}$. We see that the resultant velocity vector as measured from the radial velocity curves is directed in almost the same direction as the orbital velocity, but has a much higher value. Combined we deduce that the flow velocity of the material in the emission line region is of the order of $170 \mathrm{~km} \mathrm{~s}^{-1}$ and is directed almost in the same direction as the orbital velocity. If the gas in the emission region is connected to the gas flow in the accretion disk, we would have expected a more or less Keplerian flow with a velocity of the order of $\sim 500 \mathrm{~km} \mathrm{~s}^{-1}$. We can see in Fig. 15 that both the direction and the magnitude of the Keplerian velocity vector are very different from those observed. We therefore conclude that the gas at the emission site is decoupled from the gas in the accretion disk.

Note also that a disk overflow component, directed in the direction of the continuation of the accretion stream (roughly directed towards $\varphi \sim 0.6$ ), will not be enough to explain the resultant $v_{\text {flow }}$ component.

\subsection{The hot spot structure}

The radial velocity curves as well as the light curves of the higher Balmer lines point towards the fact that these lines are formed in the vicinity of the hot spot. The correct hot-spot structure will have to combine four results into one picture: the continuum temperature $T_{\text {spot }}$, the absorption line temperature $T_{\text {abs }}$, the $\mathrm{V}$-shaped line light curves and the U-shaped line light curves of the absorption line spectrum.

We can reconcile these by adopting a hot-spot structure that consists of two components: a photospheric component with $T_{\text {spot }}$ and a chromospheric component with

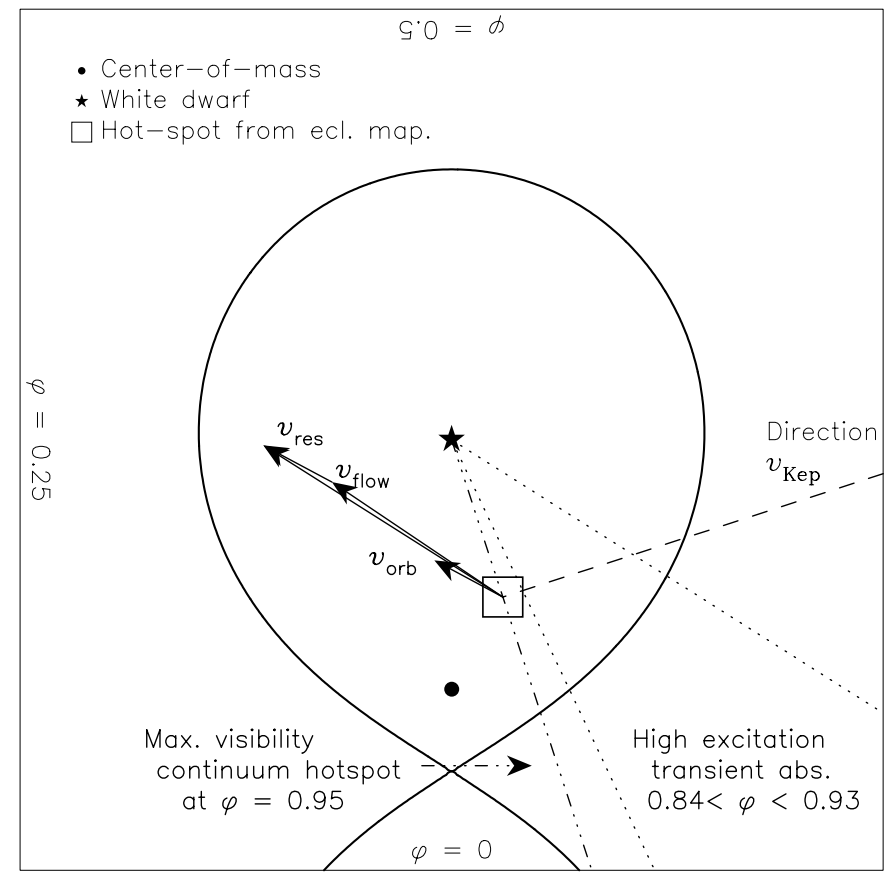

Fig. 15. A schematic view of the Roche lobe of the white dwarf in SW Sex. The orbital velocity, $v_{\text {orb }}$, at the hot spot and the observed velocity vector, $v_{\text {res }}$, are almost parallel. The gas flow velocity vector $v_{\text {flow }}$, deduced from this is $\sim 170 \mathrm{~km} \mathrm{~s}^{-1}$

$T_{\text {abs }}$. The photospheric component is responsible for the continuum radiation as derived from spectral eclipse mapping, but is also responsible for the $\mathrm{V}$-shaped light curves of the lower Balmer series. The strength of the Balmer absorption lines in a stellar spectrum reaches a maximum in late B, early A-type stars. The hot-spot spectrum will therefore contain deep photospheric Balmer absorption lines. The strength of these lines in the observed spectrum will depend on our viewing angle towards the hot-spot: coming into view at $\varphi \sim 0.7$, reaching a maximum around $\varphi \sim 0.95$ and disappearing from view again at $\varphi \sim 0.2$. Since at the same time there is a more extended region that produces Balmer line emission, and which is to first order independent on phase, we see the effect of the hot spot absorption lines as a decrease in emission in the line light curve.

It can also be seen in Fig. 10 that the amount of emission actually increases again during primary eclipse (see the excess emission with respect to the fit in $\mathrm{H} \beta, \mathrm{H} \gamma$ and $\mathrm{H} \delta$ ). This is due to the photospheric site (the hot spot) being eclipsed more than the emission-line site. We conclude that the emission lines are formed in an extended halo around the hot-spot region.

The observed absorption-line spectrum is only visible during the phase interval $0.82<\varphi<0.94$ and displays the more U-shaped light curve (Fig. 13). This component is caused by veiling by material above the hot spot (the chromospheric component with temperature $T_{\text {abs }}$ ) of continuum radiation from the hot innermost parts of the accretion disk. Only during the phase interval stated above is this hot spot chromosphere "back-lit" by radiation that 


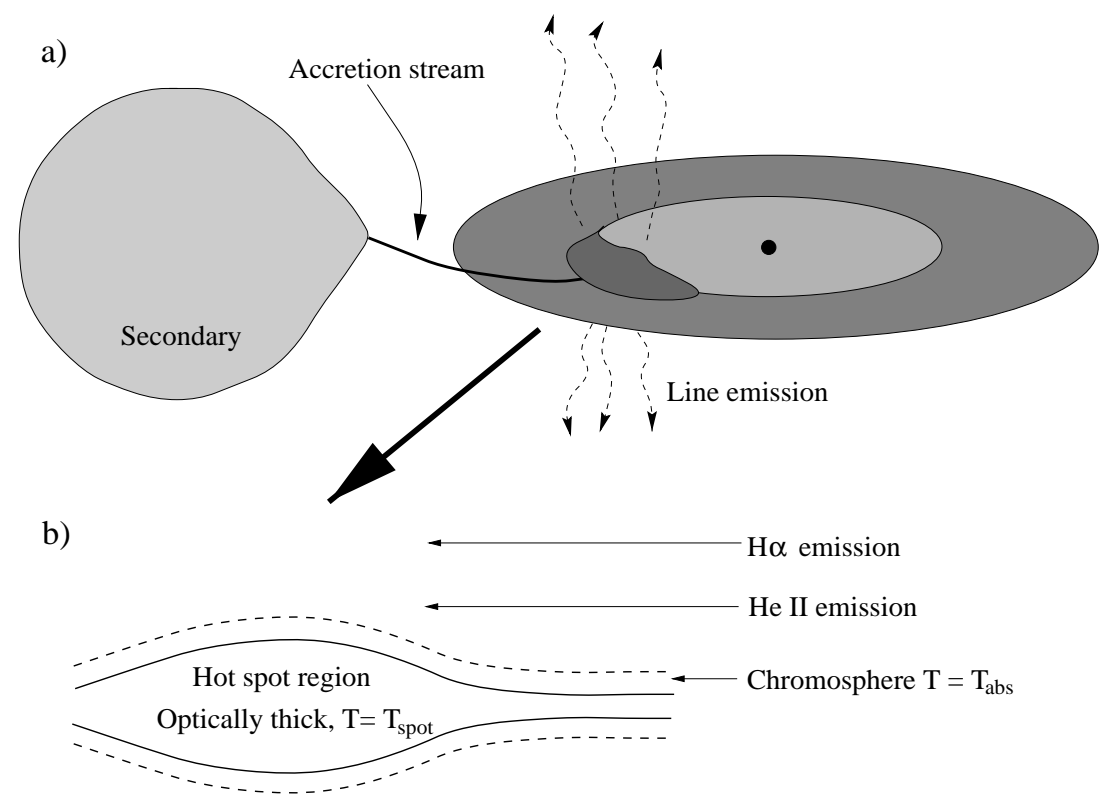

Fig. 16. Schematic view of SW Sex (top) and its hot-spot region (bottom)

is emitted by regions that are hot enough to cause the gas to show up in absorption. The sharp begin of the absorption shows that this hot region is very small itself (most likely only the white dwarf and very direct surroundings). At $\varphi=0.94$ the eclipse by the secondary star terminates our line of sight towards this hot-spot chromosphere.

\subsection{The structure of SW Sex}

The results from the spectral eclipse mapping and hot spot structure derived above combines into the following picture of SW Sex (see also Fig. 16): the accretion disk is in a quasi steady state with mass transfer rate, $\sim 10^{-9} M_{\odot} / \mathrm{yr}$, in the outer parts, but the temperature profile flattens inside a radius of $\sim 0.5 \quad R_{\mathrm{L}_{1}}$. The outer part of the disk is dominated by the hot spot region, which is also the center of a larger, vertically extended, region which is the emission site of the main emission lines, as evidenced by the phase lags and radial velocity curves of the main S-wave components in $\mathrm{H} \alpha$ and HeII $\lambda 4686$.

What is causing both the temperature disturbance in the inner disk and the extended hot-spot halo is unclear. We view it as outside the scope of the current paper to go into details on the possibilities. We would, however, like to point out a resemblance of the characteristics of SW Sex as discussed here with the old nova V Per, and we will shortly discuss the possibility of SW Sex stars being intermediate polars.

\subsection{Per: A twin to SW Sex?}

A very interesting case and a possible twin of SW Sex is the little studied old nova V Per. Shafter \& Abbott (1989) have shown that this is an eclipsing $\mathrm{CV}$ in the period gap $\left(P_{\text {orb }}=2.57 \mathrm{hr}\right)$, that shows single peaked emission lines, and most interesting, HeII $\lambda 4686$ (with a strength $>\mathrm{H} \beta$ ), and HeII $\lambda 5411$ in emission; two characteristics that are also seen in our data of SW Sex. Since, to our knowledge, no phase-resolved spectra of V Per have been published, nothing is known of the relative phasing of its emission lines with respect to the continuum eclipses. However, Wood et al. (1992) have shown from broad-band eclipse light curves, that the disk temperature profile is very similar to that found here for SW Sex. Wood et al. explain this behaviour by the absence of the inner part of the accretion disk and speculate that this could be the result of a magnetic field, which would make V Per a possible intermediate polar. A phase-resolved spectroscopic study of this system is required to understand the nature of this system and its relation to the subclass of SW Sex stars. If it is found to show the "classic" SW Sex phenomena (phase lags, transient absorption), it would extend the orbital range of SW Sex stars considerably downwards, and if V795 Her (Casares et al. 1996) is indeed a SW Sex star, be the second SW Sex star in the period gap.

\subsection{SW Sex stars as intermediate polars?}

It has been suggested that SW Sex stars (and also V Per) are intermediate polars at high inclination. It is clear that we need a source of high energy photons in SW Sex to explain the strength of the HeII $\lambda 4686$, HeII $\lambda 5411$ and CIV $\lambda 5808$ lines, and the weakness of the HeI lines in our spectrum of SW Sex. Although this source of high energy photons could be a magnetic white dwarf, these line strengths are also the only suggestions for a magnetic white dwarf. No periodic oscillations, as seen in almost all polars and intermediate polars, are found in the optical in any of the SW Sex stars. Hard X-ray emission is not detected from SW Sex stars, no cyclotron features have been observed and in V1315 Aql no polarization is detected (Dhillon \& Rutten 1995). Although none of these 
are clearly arguing against a magnetic white dwarf (since exceptions in magnetic systems can be found for all of them), the lack of any of the characteristics leaves the question of the source of X-ray flux open. Above all, we have seen that the emission site of the HeII $\lambda 4686$ radiation is not connected to the white dwarf, but to the hotspot region. We conclude that although there is not much support for a magnetic white dwarf as the source of highenergy photons, we can also not rule this option out on the basis of the current evidence.

\subsection{The SW Sex phenomenon explained (?)}

The structure of SW Sex as sketched above is capable of explaining many of the classical SW Sex phenomena as we will outline here:

- Single peaked emission lines. We have shown that the emission lines are formed at the hot-spot location. Since they are formed in a single region the lines will also be single peaked;

- Large phase shifts. The large phase shifts are naturally explained by the origin of the lines in the hot-spot region. The precise location of this region with respect to the center-of-mass will determine the phase-shift observed in the emission lines, and can in principle change with epoch;

- Phase 0.5 absorption. Although this absorption is not detected in our current observations it is relatively easy to imagine that the absorption is due to back-lighting of material overflowing the hot-spot region by the hotspot continuum radiation as seen at $\varphi \sim 0.5$. If the mass-transfer rate from the secondary is larger than observed here for SW Sex it is well possible that a larger amount of the mass is transferred to the inner disk which may cause the veiling. Back-lighting by the hot-spot continuum radiation certainly explains the phase-dependence of the absorption features which have been shown to have maximum depth around $\varphi \sim 0.45$, exactly when we see the hot-spot region from across the disk (Szkody \& Piché 1990);

- Shallow eclipses of the emission lines. Since the emission lines are formed above the disk, they will show eclipses that are less deep than that of the continuum.

Classically the SW Sex phenomenon was constrained to eclipsing systems in the period range between 3 and 4 hours. Over the last few years however, several systems at lower inclination or outside the $3-4 \mathrm{hr}$ orbital period range have been proposed as SW Sex stars, e.g. V795 Her (Casares et al. 1996), LS Pegasi (Martínez-Pais et al. 1999; Taylor et al. 1999), WX Arietis (Hellier et al. 1994), BT Mon (Smith et al. 1998) and V Per (this work). The scenario as given above does not depend on the inclination angle, although we expect that at very low inclination angles the transient absorption will become less strong.
At non-eclipsing inclination angles the phase 0.5 absorption will be caused by wind material that is back-lit by either the hot spot, or the outer disk region behind the hot spot, closer to the secondary. If the scenario outlined above holds true we expect that the velocities at which the absorption components are found will become larger with lower inclination, simply because we are looking more and more directly into the wind.

The attractiveness of this scenario over the others mentioned in Sect. 1 is the fact that it is not only able to explain all the observed feature of SW Sex stars, but especially the V-shaped Balmer emission line light curves, which cannot be explained by any of the other models.

Acknowledgements. PJG is supported by the NWO Spinoza grant 08-0 to E. P. J. van den Heuvel and a CfA fellowship. PJG wishes to thank the hospitality of Claudio Moreno and the staff of the ING observatory during a number of visits. The INT is operated on the Observatorio del Roque de los Muchachos on the island of La Palma on behalf of the British PPARC and the Dutch NWO.

\section{References}

Ashoka, B. N., Seetha, S., Marar, T. M. K., et al. 1994, A\&A, 283,455

Casares, J., Martínez-Pais, I. G., Marsh, T. R., Charles, P. A., \& Lazaro, C. 1996, MNRAS, 278, 219

Dhillon, V. S., Marsh, T. R., \& Jones, D. H. P. 1997, MNRAS, 291,694

Dhillon, V. S., \& Rutten, R. G. M. 1995, MNRAS, 277, 770

Frank, J., King, A. R., Raine 1992, in Accretion Power in Astrophysics, Cambridge Astrophysics Series No. 21, CUP, Cambridge UK

Green, R. F., Ferguson, D. H., Liebert, J., \& Schmidt, M. 1982, PASP, 94, 560

Green, R. F., Schmidt, M., \& Liebert, J. 1986, ApJS, 61, 305

Gull, S. F., \& Skilling, J. 1983, in Indirect Imaging, Measurement and Precessing for Indirect Imaging, ed. J. A. Roberts (Cambridge University Press, Cambridge, UK)

Hellier, C., \& Robinson, E. L. 1994, ApJ, 431, L107

Hellier, C., Ringwald, F. A., \& Robinson, E. L. 1994, A\&A, 289,184

Hellier, C. 1996, ApJ, 471, 949

Hellier, C. 1998, PASP, 110, 420

Horne, K. 1985, MNRAS, 213, 129

Horne, K. 1986, PASP, 98, 609

Horne, K. 1999 [astroph-9901007]

Honeycutt, R. K., Schlegel, E. M., \& Kaitchuck, R. H. 1986, ApJ, 302, 388

Jacoby, G. H., Hunter, D. A., \& Christian, C. A. 1984, ApJS, 56,257

Marsh, T. R. 1988, MNRAS, 231, 1117

Martínez-Pais, I. G., Rodríguez-Gil, P., \& Casares, J. 1999, MNRAS, 305, 661

Massey, P., Strobel, K., Barnes, J. V., \& Anderson, E. 1988, ApJ, 328, 315

Patterson, J. 1984, ApJS, 54, 443

Penning, W. R., Ferguson, D. H., McGraw, J. T., Lienert, J., \& Green, R. F. 1984, ApJ, 276, 233 
Rutten, R. G. M., Van Paradijs, J., \& Tinbergen, J. 1992, A\&A, 260, 213

Rutten, R. G. M., Dhillon, V. S., Horne, K., Kuulkers, E., \& Van Paradijs, J. 1993, Nature, 362, 518

Rutten, R. G. M., Dhillon, V. S., Horne, K., \& Kuulkers, E. 1994, A\&A, 283, 441

Rutten, R. G. M. 1998, A\&AS, 127, 581

Shafter, A. W., \& Abbott, T. M. C. 1989, ApJ, 339, L75

Szkody, P., \& Piché, F. 1990, ApJ, 361, 235

Smak, J. 1994, Acta Astr., 44, 265

Smith, D. A., Dhillon, V. S., \& Marsh, T. R. 1998, MNRAS, 296,465
Taylor, C. J., Thorstensen, J. R., \& Patterson, J. 1999, PASP, 111,184

Thorstensen, J. R., Ringwald, F. A., Wade, R. A., Schmidt, G. D., \& Norsworthy, J. E. 1991, AJ, 102, 272

Van Kerkwijk, M. H., Van Paradijs, J., Zuiderwijk, E. J., et al. 1995, A\&A, 303, 483

Warner, B. 1995, Cataclysmic Variable Stars, Cambridge Astrophysics Series 28, CUP, Cambridge, UK

Williams, R. E. 1989, AJ, 97, 1752

Wood, J. H, Abbott, T. M. C., \& Shafter, A. W. 1992, ApJ, 393,729 